\title{
Von der „Islampolitik“ zum „Dialog mit der islamischen Welt" - Anmerkungen zum Verhältnis zwischen Orientalistik und deutscher (Außen-)politik
}

TIM EPKENHANS (BISCHKEK)

\begin{abstract}
„Da ist es denn eine der Grundfragen, wie man sich zum Islam überhaupt stellt, ob man ihn bekämpft, befördert oder indifferent behandelt $[\ldots]$ ob man sich in die islamischen Verhältnisse einmischen soll, oder ob man die europäische Staatsleitung vollkommen trennen soll von dem islamischreligiösen Leben der Bevölkerung, um sich höchstens auf ein allgemeines Aufsichtsrecht zu beschränken.“ Carl Heinrich Becker (1915) ${ }^{1}$
\end{abstract}

\section{Einleitung}

Als ich Anfang der 1990er Jahre mein Studium der Islamwissenschaft und Iranistik aufnahm, reagierten Verwandte und Freunde eher mit Unverständnis auf meine Entscheidung, häufig mit dem Hinweis, dass es sich doch wohl um eine brotlose Kunst mit geringen beruflichen Perspektiven handle. Das Interesse am ,Islam` war in der deutschen Öffentlichkeit und Politik marginal und die deutsche Orientalistik forschte und publizierte weitgehend selbstgenügsam in einem kommoden Elfenbeinturm. ,Den Islam‘ erklärten der deutschen Öffentlichkeit (und Politik) zumeist selbsternannte Experten (wie etwa der notorische Peter Scholl-Latour), die in öffentlichen Debatten Fachvertretern der Orientalistik regelmäßig den Rang abliefen (und heute noch ablaufen) und sich weitaus effizienter den spezifischen Regeln einer Mediengesellschaft unterwarfen. ${ }^{2}$ Offensicht-

1 Carl Heinrich Becker: „Islampolitik“, in: Die Welt des Islams 3 (1916), S. 101-120, hier S. 108.

2 Vgl. hier etwa die TV-Debatten während des Golfkrieges 1991. Allerdings ist hier anzumerken, dass sich Vertreter der orientalistischen Fachdisziplinen durchaus kritisch gegen Scholl-Latour zu Wort meldeten (vgl. Verena Klemm/Karin Hörner (Hg.), Das Schwert des Experten - Peter Scholl-Latours verzerrtes Araber- und Islambild, Heidelberg: Palmyra 1993). Gernot Rotter hatte zuvor bereits nachgewie- 
lich war und ist es vielen orientalistischen Fachvertretern unangenehm, sich in die profanen Untiefen deutscher Medienöffentlichkeit oder gar der Politikberatung zu begeben. Das Unbehagen in der Islamwissenschaft offenbarte sich nicht zuletzt in der Kontroverse um die Verleihung des Friedenspreises des deutschen Buchhandels 1995 an die Grand Old Dame der deutschen Orientalistik Annemarie Schimmel. Um es mit Stefan Wild zu sagen: „Ein Fach verkriecht sich“33.

Der 11. September 2001 und die folgenden Ereignisse insbesondere in Europa hatten schließlich zur Folge, dass ,Islam' zu einem der zentralen Themen in der deutschen Öffentlichkeit und Politik avancierte. Mittlerweile bemüht sich eine Flut von einschlägigen Publikationen um eine Erklärung des ,Islam` und den Absolventen der orientalistischen Disziplinen eröffneten sich zahlreiche berufliche Perspektiven unter anderem in staatlichen Institutionen, wie etwa dem Verfassungsschutz, Bundesnachrichtendienst und dem Auswärtigen Amt. Letzteres initiierte bereits Ende 2001 eine umfangreiche diplomatische Initiative unter dem Titel „Dialog mit der islamischen Welt“ (oder in der Kurzform „Islamdialog“), zu dessen Implementierung auch Orientalisten als Referenten auf befristete Stellen vergleichbar zum höheren Dienst rekrutiert wurden. ${ }^{4}$ Auch der Verfasser dieser Zeilen fand sich - durchaus zu seiner Überraschung - nach seiner Promotion im Frühjahr 2002 als so genannter ,Islambeobachter' an der deutschen Botschaft in Duschanbe, Tadschikistan, wieder - doch dazu später mehr.

Die Anwerbung von Orientalisten im Rahmen einer dezidiert auf die islamische Welt fokussierten Außenpolitik mag unter aufmerksamen Beobachtern der deutschen Orientalistik unangenehme Assoziationen mit einer Zeit evozieren, wo Fachvertreter und dilettantische Laien schon einmal versucht hatten, die deutsche ,Islampolitik‘ zu beeinflussen.

sen, dass Gerhard Konzelmann (Publizist und Nahost-Korrespondent der ARD) in seinem zahlreichen Publikationen wissenschaftliche Arbeiten plagiiert hatte (vgl. Gernot Rotter: Allahs Plagiator. Die publizistischen Raubzüge des „Nahostexperten“ Gerhard Konzelmann, Heidelberg: Palmyra 1992). Dennoch ist Scholl-Latour noch heute einer der erfolgreichsten Sachbuchautoren in Deutschland und auch Konzelmann publiziert weiterhin (wenn auch weniger erfolgreich).

3 Stefan Wild: „Der Friedenspreis und Annemarie Schimmel: Eine Nachlese“, in: Die Welt des Islams 36 (1996), S. 107-122, hier S. 121.

4 Die Einrichtung befristeter Stellen für bestimmte Aufgaben war bis zu diesem Zeitpunkt im Auswärtigen Amt gänzlich unüblich. Seit Einrichtung des AA 1951 wurden gelegentlich auch Absolventen der orientalistischen Fächer in den auswärtigen Dienst berufen. Der auswärtige Dienst untersteht jedoch dem Paradigma des Generalismus, d.h. die fachliche Qualifikation eines Beamten im Auswärtigen Dienst wird nur bedingt in der Stellenplanung berücksichtigt, wobei die amtsinterne Attaché-Ausbildung eine Verwendung in allen Abteilungen des AA gewährleisten soll. 


\section{Der Erste Weltkrieg und die „Revolutionierung“ der islamischen Welt}

Nach der Reichsgründung 1871 und insbesondere während der Regentschaft Wilhelm II. verfolgte das Deutsche Reich eine ambitionierte Außenpolitik, die ihm einen Platz an der Sonne in Form überseeischer Kolonien garantieren sollte. Weder die ,Weltpolitik' von Reichskanzler von Bülow noch die heterogenen Vorstellungen verschiedener Lobbyistengruppen (wie etwa dem Alldeutschen Verband) folgten konsistenten Ideen, wie die koloniale Expansion im Orient und insbesondere die Politik gegenüber dem Osmanischen Reich ausfallen sollte, führte aber zu zunehmenden Rivalitäten mit Russland, Großbritannien und Frankreich. Fachvertreter der orientalistischen Disziplinen, etwa Carl Heinrich Becker, Eugen Mittwoch oder Martin Hartmann, bemühten sich nachhaltig um eine aktive Mitgestaltung der ,Orientpolitik' des Deutschen Reiches bereits im Vorfeld des Ersten Weltkrieges. Obwohl es vor allem ,Laienorientalisten“ wie etwa Max von Oppenheim ${ }^{5}$ oder Konsul Schabinger waren, die sich aktiv um eine „Revolutionierung der islamischen Gebiete unserer Feinde "6 bemühten, hatten sich auch zahlreiche Fachvertreter deutscher Universitäten nur allzu bereitwillig für Pläne der Reichsleitung und des Auswärtigen Amtes einspannen lassen. Dies ist mittlerweile detailliert und kritisch dokumentiert worden. ${ }^{7}$ Allerdings, das En-

5 Max von Oppenheims Karriere war ungewöhnlich für einen Nachkommen der vermögenden Bankiersfamilie Oppenheim. 1860 geboren, trat er 1883 nach seinem Jura-Studium in den preußischen Verwaltungsdienst ein. Das väterliche Vermögen erlaubte ihm bereits frühzeitig die Möglichkeit ausgedehnter Reisen nach Nordafrika und Indien. Oppenheim nutzte einen längeren Aufenthalt in Alexandria, um auf den Spuren des berühmten Richard Francis Burton ,ein Orientale' zu werden. Zwischen 1896 und 1910 residierte Oppenheim als Attaché an der deutschen Gesandtschaft in Kairo. Seine nachrichtendienstliche Funktion verpflichtete ihn zu zahlreichen Reisen im Gebiet der Arabischen Halbinsel und der Levante, wo er 1899 die hethitische Siedlung Tell Halaf (im heutigen Syrien) entdeckte. 1910 quittierte er den diplomatischen Dienst und leitete bis 1913 die archäologischen Ausgrabungen in Tell Halaf. Kurz nach Ausbruch des Ersten Weltkrieges berief das Auswärtige Amt Oppenheim zur Bearbeitung der Angelegenheiten die islamische Welt betreffend zurück. Vgl. R. L. Melka: „Max Freiherr von Oppenheim. Sixty Years of Scholarship and Political Intrigue in the Middle East“, in: Middle Eastern Studies 9 (1973), S. 81-93.

6 So der Titel der Denkschrift (1914) Max von Oppenheims. Siehe hierzu die Edition von Tim Epkenhans: „Geld darf keine Rolle spielen“, Teil 1 und Teil 2, in: Archivum Ottomanicum 18 (2000), S. 247-250 und 19 (2001), S. 121-163.

7 Vgl. etwa: Gottfried Hagen: „German Heralds of Holy War: Orientalists and Applied Oriental Studies“, in: Comparative Studies of South Asia, Africa and the Middle East 24 (2004), S. 145-162; Ludmila Hanisch: „Gelehrtenselbstverständnis, wissenschaftliche Rationalität und politische ,Emotionen“: Ein Nachtrag“, in: Die Welt des Islams 32 (1992), S. 107-123; Peter Heine: „C. Snouck Hrugronje versus C. H. Becker. Ein Beitrag zur Geschichte der angewandten Orientalistik“, in: Die Welt des Islams 23 (1984), S. 378-387; Martin Kröger: „Revolution als Programm. Ziele und Realität deutscher Orientpolitik im Ersten Weltkrieg“, in: Wolfgang Michalka (Hg), Der Erste Weltkrieg. Wirkung, Wahrnehmung, Analyse, München, 
gagement von Orientalisten in der Politik ist keineswegs ein deutsches Phänomen: Der eminente russische Orientalist Vitaly V. Barthold wirkte aktiv an der stalinistischen Nationalitätenpolitik in Zentralasien mit, Snouck Hurgronje war in der niederländischen Kolonialverwaltung Indonesiens tätig und die Zahl britischer Orientalisten, die zwischen militärisch-administrativer und akademischer Karriere oszillierten, ist beträchtlich - in den Worten Disraelis: The East was a career. $^{8}$

Carl Heinrich Becker äußerte sich 1904 noch mit deutlichem Unbehagen ge-

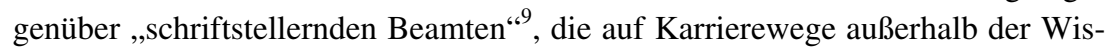
senschaft als Diplomaten oder Verwaltungsbeamten zurückblickten und sich dennoch zu orientalistischen Themen zumeist in einem politischen Kontext äuBerten (wie etwa Max von Oppenheim). Dies soll jedoch nicht bedeuten, dass Becker eine Einmischung in das politische Feld a priori als unwissenschaftlich ablehnte. Im Gegenteil, ab 1908 leitete er das Seminar für Geschichte und Kultur des Vorderen Orients in Hamburg, welches auch mit kolonialpolitischen Funktionen betraut war und äußerte sich - wie das einleitende Zitat zeigt - wiederholt zu dem Themenkomplex Islam und koloniale Politik. Beckers Mahnung, eine

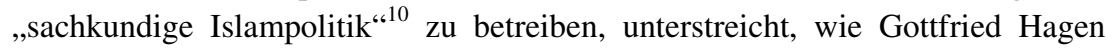
treffend bemerkt, sein Bestreben, ein ,,academic monopoly for authorative interventions in the public sphere ${ }^{\text {"11 }} \mathrm{zu}$ verteidigen. Im Kontext des Gelehrtenselbstverständnisses des späten Kaiserreiches rangierten Fragen der Diskurshoheit vermutlich vor Fragen der Relevanz oder praktischen Anwendbarkeit der Orientalistik.

Der Ausbruch des Ersten Weltkrieges und die Einrichtung der Nachrichtenstelle für den Orient durch das Auswärtige Amt gab zahlreichen Orientalisten (aber vor allem auch ,schriftstellernden Beamten“, Dilettanten und gewitzten Betrügern $^{12}$ ) die Gelegenheit, Politik und Form der deutschen ,Orientpolitik` zu gestalten. Die deutsche Begeisterung für die Ausrufung des ğihād gegen die Alliierten durch Mehmet V. am 14. November 1914 verdeutlicht dies und ist zudem Kern einer emotionalen Debatte zwischen Becker und seinem niederländischen Kollegen Snouck Hurgronje. Dieser griff die deutsche Orientpolitik und Becker, der den Aufruf zum ǧihād ausdrücklich unterstützte, in seinem Artikel „Heiliger Krieg made in Germany“ scharf an. ${ }^{13}$ Der Austausch zwischen Becker und Hur-

Zürich: Piper 1994, S. 366-391; Herbert L. Müller: Islam, Ğihād („Heiliger Krieg“) und das Deutsche Reich, Frankfurt/M u.a.: Lang 1991.

8 Vgl. Edward Said: Orientalism, London u.a.: Penguin Books 2003 (Erstausgabe 1978), S. 5.

9 Zitiert nach L. Hanisch, Gelehrtenselbstverständnis, S. 111.

10 Ebd., S. 107.

11 G. Hagen: Applied Oriental Studies, S. 154.

12 Vgl. M. Kröger: Orientpolitik, S. 376f.

13 Vgl. P. Heine: Hurgronje versus C. H. Becker, S. 379f.; Hurgronje verfasste 1915 einen Artikel mit dem Titel „Heilige Oorlog made in Germany“. 
gronje wirft weniger Fragen der wissenschaftlichen Ethik auf, sondern beleuchtet vielmehr Elemente des orientalistischen Diskurses jener Zeit. ${ }^{14}$ Auch wenn verschiedene Auffassungen über das vermeintlich fanatische Potential des ,Islam“ unter deutschen Orientalisten bestanden, unterstreicht das folgende Zitat von Martin Hartmann wesentliche Elemente des Diskurses:

„Es sei hiermit gewarnt, durch Erregung des religiösen Fanatismus Unruhen herbeizuführen. Gegen einen solchen Versuch würden alle Kulturstaaten einmütig zusammenstehen [...]. Islam ist eine Religion von $\mathrm{Haß}$ und Krieg. Es darf nicht das herrschende Prinzip in einer Nation der zivilisierten Welt werden. “15

Dass auch die ,sachkundige Islampolitik“ eines Carl Heinrich Becker nicht die erwünschten Erfolge zeitigte, belegt die Einschätzung eines weiteren deutschen Orientalisten, Friedrich Rosen (der in der Weimarer Republik kurzzeitig Außenminister werden sollte), der weitaus nüchterner und ohne Verweise auf die vermeintlichen kulturellen Dispositionen ,der Muslime‘ die deutschen Ambitionen als ,Wahnvorstellungen' charakterisiert und feststellt:

„,[]m Auswärtigen Amt glaubte man damals ebenso fest an die grüne Fahne des Propheten und den Heiligen Krieg, wie einige Jahre später an den Völkerbund und die Segnungen der Locarno-Politik. [...] So wie Voltaire als der Vater der französischen Revolution angesehen wird, so konnte man Karl May als den Vater unserer Orientpolitik dieser Zeit betrachten." ${ }^{16}$

\section{Eine Frage der Qualität oder der Ethik?}

Peter Heine schließt seinen Beitrag zur Auseinandersetzung zwischen Becker und Hurgronje, indem er die Frage der Verantwortung einer ,,anwendungsbezogenen Wissenschaft" aufwirft:

„Die Gefahr, in die jede angewandte Orientalistik geraten kann, die Gefahr nämlich, in direkte politische Auseinandersetzungen zu geraten. In solchen Auseinandersetzungen geht es dann um wissenschaftliche Fragen eher am Rande. Solch eine Situation kann auf Kosten der Qualität gehen. [...] Gerade in einer Zeit, in der viele Orientalisten in den Bereichen der angewandten Orientalistik ein berufliches Betätigungsfeld sehen oder sehen müssen, sollte die Kontroverse Snouck Hurgronje/Becker uns ein warnender Hinweis auf die hohe Verantwortung jeder anwendungsbezogenen Wissenschaft sein.“17

14 Said behandelt die deutsche Orientalistik nur am Rande, verweist aber mehrfach auf Hurgronje (vgl. Orientalism, S. 255f.).

15 Vgl. Martin Hartmann: „Das Ultimatum des Panislamismus“, in: Das freie Wort 11 (1911), S. 605-610, hier S. 606.

16 Friedrich Rosen: Aus einem diplomatischen Wanderleben, Bd. 3-4, Wiesbaden: Limes Verlag 1959, S. 54f.

17 P. Heine: Hurgronje versus C. H. Becker, S. 387. 
Obwohl Heine hier implizit eine ethische Dimension aufwirft, stellt sich ihm jedoch vordringlich das Problem des Qualitätsverlustes im Falle einer politischen Kontamination, also um eine eher generelle Ablehnung, dem Fach eine politische Dimension bzw. Funktion zuzuschreiben. Auch Hanisch erwähnt in ihrer Reaktion auf Heine die Webersche Verantwortungsethik im Kontext der gesellschaftlichen Verantwortung innerhalb eines politischen Systems. ${ }^{18}$ Wie Hagen bereits angemerkt hat, verweisen Hanisch und Heine auf die generelle Problematik des Verhältnisses zwischen Wissenschaft und gesellschaftlicher Verantwortung, ohne die spezifischen Charakteristika der Orientalistik zu berücksichtigen, d.h. die diskursive Ethik, die Said in Orientalism thematisiert. ${ }^{19}$

Die Frage der gesellschaftlichen Verantwortung von Wissenschaft ist vor allem dann von erheblicher Relevanz, wenn das politische und gesellschaftliche System, in dem Wissenschaft praktiziert wird, totalitär ist, wie etwa während der NS-Diktatur. Die deutsche Orientalistik hat sich in den vergangenen Dekaden zwar detailliert mit der Rezeption des Faschismus/Nationalsozialismus in der islamischen Welt beschäftigt ${ }^{20}$, aber eine erste ausführlichere Einordnung der Orientalistik in die Wissenschaftsgeschichte des NS-Regimes ließ bis 2006 auf sich warten. ${ }^{21}$ Die zögerliche Aufarbeitung könnte dadurch bedingt sein, dass nicht wenige eminente Orientalisten während der NS-Zeit ihre akademische Karriere begannen und nach dem Zweiten Weltkrieg fortsetzten. Auch zeigt Ellinger in seiner Dissertation auf, dass Orientalisten als Funktionsträger im NS-System agierten. Das Scheitern der Wahrnehmung sozialer Verantwortung von Wissenschaft während der nationalsozialistischen Diktatur mit ihren teils wissenschaftlich legitimierten Perversionen von Rassenauslese ist selbstverständlich kein genuines Problem der Orientalistik, sondern aller Disziplinen, aber wohl mit ein Grund für den Rückzug der Orientalistik in einen wissenschaftlichen Elfenbeinturm und für das große Unbehagen gegenüber einer, angewandten Wissenschaft ${ }^{*}$ nach dem Zweiten Weltkrieg. Ebenfalls ist zu bedenken, dass sich der gesellschaftspolitische Rahmen der Orientalistik in beiden deutschen Staaten veränderte. Die westdeutsche (und bedingt auch die ostdeutsche) Außenpolitik stand unter den Prämissen der deutschen Teilung (Hallstein-Doktrin) und der Konsolidierung der BRD in Europa. Der außenpolitische Diskurs wurde nicht mehr vordringlich im Kontext von nationalstaatlichen Interessen und Zielen formuliert, sondern wechselte auf eine multilaterale (EU und Vereinte Nationen) sowie abstrakte

18 L. Hanisch: Gelehrtenselbstverständnis, S. 107 und 123f.

19 Vgl. G. Hagen: Applied Oriental Studies, S. 155.

20 Vgl. etwa Stefan Wild: „National Socialism in the Arab near East between 1933 and 1939“, in: Die Welt des Islams 25 (1985), S. 126-173.

21 Vgl. Ekkehard Ellinger: Deutsche Orientalistik zur Zeit des Nationalsozialismus 1933-1945, Edingen-Neckarshausen: deux-mondes-Verlag 2006. Siehe auch K. Kreiser: Gotthard Jäschke, S. 406-423. 
Ebene, in der Demokratisierung, Menschenrechte und wirtschaftlich-soziale Entwicklung zentrale Positionen einnahmen. ,Islam“ oder gar eine ,Islampolitik“ stand nicht auf der Agenda deutscher Außenpolitik, zumal ein Großteil der islamischen Welt durch säkulare Regime beherrscht wurde. ,Islam“ erlangte erst wieder mit der Revolution in Iran 1979 und der graduell wachsenden Bedeutung islamischer politischer Gruppen in den 1980er und 1990er Jahren an Bedeutung.

Edward Saids Orientalism erschütterte zwar die Selbstgenügsamkeit der deutschen orientalistischen Forschung und förderte eine anhaltende Diskussion ${ }^{22}$, aber es scheint mir, dass andere Disziplinen die Anregungen und Überlegungen Saids weitaus bereitwilliger aufnahmen:

„Indem die Islamwissenschaft den allgemeinen sozial- und mehr noch den kulturwissenschaftlichen Diskurs weitgehend ignorierte, isolierte sie sich und wurde für andere Forscher, die sich mit verwandten Themen in anderen Sprachen beschäftigen, unattraktiv, häufig unlesbar.“23

\section{Orientalistik nach dem 11. September - Islamdialog und auswärtige Politik}

Die Bundesregierung reagierte nach dem 11. September 2001 umgehend mit der Verabschiedung der so genannten Anti-Terror-Pakete (ATP) I (noch im September 2001) und II (Dezember 2001), die nicht nur eine Reihe von Gesetzesänderungen beinhalteten ${ }^{24}$, sondern verschiedenen Ministerien und Bundesbehörden zusätzliche Haushaltsmittel in Höhe von 1,5 Mrd. Euro bewilligten.

Auch das Auswärtige Amt wurde im Rahmen der ATP mit zusätzlichen Haushaltsmitteln bedacht, mit denen unter anderem der „Dialog mit der islamischen Welt ${ }^{\star 25}$ finanziert werden sollte (neben anderen Antiterrorismusinitiativen). Das Dialogprojekt sah vor, die Öffentlichkeitsarbeit der deutschen Auslandsvertretungen (inklusive der Goethezentren) in der islamischen Welt zu intensivieren, Islambeobachter an verschiedene Auslandsmissionen zu entsenden und ein eigenes Referat (heute 626) unter der Leitung eines Beauftragten für den Dialog mit der islamischen Welt (bis 2005 Botschafter Dr. Mulack) zu etablie-

22 Vgl. u. a. Reinhard Schulze: „Orientalistik und Orientalismus“, in: Werner Ende/Udo Steinbach (Hg), Der Islam in der Gegenwart. München: C.H. Beck 1995, S. 707-718.

23 Navid Kermani: „Zur Zukunft der Islamwissenschaft“, in: Fikrun wa Fann 81 (2005), S. 3, http://www.goethe.de/mmo/priv/1224466-STANDARD.pdf vom August 2007.

24 Vor allem die Änderungen StGB § 129a („Anti-Terrorismus-Paragraph“), Aufhebung des Religionsprivilegs im Vereinsgesetz (§ 3), Auskunftspflicht der Banken über Konten verdächtiger Organisationen.

25 Vgl. etwa: Auswärtiges Amt (Hg.), Dialog mit der islamischen Welt, Berlin 2006 (www.auswaertiges-amt.de). 
ren. ${ }^{26}$ Laut der Homepage des Auswärtigen Amtes besteht das Ziel des Dialogs darin:

,[...] die Verständigung zwischen dem ,Westen“ und der islamischen Welt sowie innergesellschaftlichen Pluralismus und den Abbau antiwestlicher Feindbilder in islamisch geprägten Gesellschaften zu fördern. Deutschland hat als erstes westliches Land bereits 2002 einen Politikschwerpunkt ,Dialog mit der islamischen Welt' (kurz: ,Islamdialog') im Auswärtigen Amt geschaffen. Unter anderem wurde das Amt des Beauftragten für den Dialog mit der islamischen Welt eingerichtet, das derzeit [2007] Botschafter HansGünter Gnodtke innehat. Er berät alle Arbeitsebenen im Auswärtigen Amt und wird von einem Arbeitsstab unterstützt, der ein Netz von sprach- und fachkundigen ReferentInnen an den deutschen Auslandsvertretungen in der islamischen Welt koordiniert. Aufgabe der ReferentInnen ist es, über die Bedeutung von Islam und Islamismus in ihren Gastländern zu berichten, sowie lokale Partner für die Zusammenarbeit zu identifizieren und mit diesen konkrete Projekte auszuarbeiten. ${ }^{، 27}$

Während ein Teil der hier erwähnten ,ReferentInnen`zeitlich befristet (für zwei Jahre) in den vergleichbaren höheren Dienst eingestellt wurden, ${ }^{28}$ erlaubten die ATP eine umfangreiche Konsolidierung des Stellenplans im Auswärtigen Amt. Auch das Auswärtige Amt war in den 1990er Jahren von der Sparpolitik der Bundesregierungen Kohl und Schröder nicht verschont geblieben, was zu sukzessiven Streichungen von Planstellen in Berlin, Bonn und an den Auslandsvertretungen führte. Insbesondere im höheren Dienst ergab sich dadurch ein erheblicher Beförderungsstau. Im Rahmen des ATP konnten erstmals wieder Planstellen in Referaten und Auslandsmissionen designiert werden, die zuvor entweder nicht besetzt oder eingerichtet waren. ${ }^{29}$ Diese Planstellen wurden sowohl für den gehobenen als auch höheren Dienst ${ }^{30}$ für bereits im auswärtigen Dienst tätige Beamte eingerichtet und hatten nicht immer einen unmittelbaren Bezug zu den ATP. Teilweise erfolgte eine Entsendung auf ,Islambeobachter'-Stellen nicht aufgrund der Qualifikation (einer der amtsinternen Islambeobachter teilte mir schmunzelnd mit, seine Qualifikation beruhe wohl auf einer Zwischenlandung in Kabul in den 1960er Jahren - eine offensichtlich hinreichende Erfahrung für die Bedingung, die islamische Welt zu kennen), sondern hatte andere Beweggründe

26 Das Referat 626 untersteht der Abteilung 6 für Kultur und Kommunikation.

27 http://www.auswaertiges-amt.de/diplo/de/Aussenpolitik/Kulturpolitik/DialogIslam/ IslamDialog.html.

28 Wie bereits erwähnt, ist diese Einstellungspraxis für das Auswärtige Amt unüblich, wird aber zurzeit (2007) in begrenztem Umfang weitergeführt.

29 Nach meinen Informationen (und durch Gespräche mit Kollegen) wurden 2002 zunächst 45 externe Referentinnen und Referenten befristet eingestellt, während etwa 200 Planstellen neu eingerichtet bzw. bestehende Planstellen ein zusätzliches Portfolio im Rahmen des ATP erhielten.

30 Für die Organisation des AA vgl. BGB1 I 1990, 1842, Gesetz über den Auswärtigen Dienst (GAD) vom 30. August 1990. 
(wie etwa der bereits erwähnte Beförderungsstau). Auch bei den externen Referentinnen und Referenten handelte es sich nicht ausschließlich um Orientalisten; Juristen, Slawisten oder Chemiker wurden ebenfalls berücksichtigt, da die ATP nicht nur ,Islambeobachtung ' umfassten, sondern auch den Komplex der ,Terrorismusbekämpfung'. Während ein Teil der Referenten in Berlin (vor allem im Islam-Referat) tätig waren, entsandte das Auswärtige Amt Referenten an verschiedene Auslandsmissionen, z.B. in Nigeria, Libanon, Iran und - in meinem Fall Tadschikistan. In der Regel, aber nicht immer, korrespondierte die Entsendung bzw. der Einsatz der Referentinnen und Referenten mit ihrer spezifischen Qualifikation (insbesondere der Sprachkompetenz). Unter welchen Gesichtspunkten das Auswärtige Amt einige der Auslandsstationen auswählte, entzieht sich jedoch meiner Kenntnis.

Neben der konkreten Islambeobachtung durch Referenten initiierte bzw. intensivierte das Auswärtige Amt ab 2002 eine Vielzahl von Dialogprojekten, insbesondere in Form von periodisch erscheinenden Publikationen, Konferenzen und Seminaren, auf die hier nicht weiter eingegangen werden soll, die aber nach wie vor einen integralen Bestandteil des ,Islamdialogs ‘ darstellen. ${ }^{31}$

\section{Islambeobachtung in Tadschikistan}

Im Nachhinein war ich mehr als (positiv) überrascht, als im Frühjahr 2002 mein Erlass für die Entsendung an die deutsche Botschaft in Duschanbe, Tadschikistan, eintraf. Zwar verfügte ich über rudimentäre Kenntnisse der Landeskunde und Sprache (d.h. des Tadschikischen, einer Form des Farsi), allerdings hatte ich keinerlei Erfahrung mit dem post-sowjetischen Raum. Trotz seiner Nachbarschaft zu Afghanistan ist Tadschikistan von eher nachrangigem Interesse für die deutsche Außenpolitik. Die 1995 eingerichtete Botschaft ist eine so genannte Kleinstvertretung mit 10 Entsandten, wobei konsularische Tätigkeit (Visa-Angelegenheiten, ${ }^{32}$ Betreuung von deutschen - teilweise auch europäischen - Bürgern in Tadschikistan sowie der deutschen Minderheit ${ }^{33}$ ), die Koordinierung der wirtschaftlichen Zusammenarbeit sowie der Bildungsinitiativen die wesentli-

31 So wurde beispielsweise eine arabische Version der Homepage des Auswärtigen Amtes eingerichtet. Siehe auch die Homepage der Online-Zeitschrift www.qantara. de und des deutschen Informationszentrums in Kairo (www.gic.de) sowie die Zeitschrift Fikrun wa Fann.

32 Die deutsche Botschaft in Duschanbe ist - wie in vielen der zentralasiatischen Staaten - die einzige Botschaft, die „Schengen“-Visa ausstellt.

33 In den 1980er Jahren lebten etwa 40.000 Deutsche (zumeist Wolga-Deutsche und deren Nachkommen, die in den 1940er Jahren deportiert wurden) in Tadschikistan, vor allem in Duschanbe. Ein Großteil der deutschen Minderheit verließ Tadschikistan während des Bürgerkrieges; vgl. Kamoludin Abdullaev/Shahram Akbarzadeh (Hg.), Historical Dictionary of Tajikistan, Lanham, MD u.a.: Scarecrow Press 2002. 
chen Aufgabengebiete darstellen. Wie die bereits zitierte Zielvorgabe des Auswärtigen Amtes verdeutlicht, fiel die Erlasslage (d.h. die amtsinterne Definition des Aufgabenbereiches) für die ,Islambeobachtung ' insgesamt vage aus und erlaubte eine recht freie Auslegung durch den Islambeobachter, aber auch durch die Vorgesetzen in der Auslandsvertretung. Zu den Kernaufgaben zählt die Berichterstattung über Ausdrucksformen islamischer Religiosität bzw. islamische Gruppierungen, Kontaktaufnahme zu Regierungsvertretern, die mit islamischen Angelegenheiten betraut sind, und mit offiziellen wie inoffiziellen islamischen Würdenträgern sowie die Identifikation von Projekten im Kontext des ,Islamdia$\operatorname{logs} .34$

Wie Kollegen aus den ATP berichteten, gelang die Integration des Islambeobachters in eine Auslandsvertretung nicht immer ohne Konflikte. Gelegentlich hatten Vorgesetzte Vorbehalte gegenüber ,amtsfremden“ Mitarbeitern, stellten sie als ,Islamisten“ vor, ignorierten die Erlasslage oder standen dem Dialogprojekt kritisch gegenüber. Die vage Erlasslage ergab zudem, dass die Berichterstattung der verschiedenen Islambeobachter kaum einheitlichen Kriterien folgte: Akademische Interessen sowie Vorbildung eines Islambeobachters konnten somit leicht Themen der Berichterstattung beeinflussen. Details bestimmter regionaler Auslegungen islamischer Rechtsvorstellungen füllten umfangreiche Berichte, deren Relevanz für Laien nicht immer nachvollziehbar war. Bei der Identifikation geeigneter Projekte im Rahmen des Islamdialogs ergaben sich ebenfalls Probleme durch die wenig konzisen Vorgaben, wie solche Projekte auszusehen hätten. Eine Haushaltssperre 2003 erschwerte zudem die Realisierung von relevanten Projekten, da nun Mittel nur noch bedingt zur Verfügung standen.

In meinem Fall hatte ich außerordentliches Glück, an eine Auslandsvertretung zu kommen, deren Mitarbeiter mich hervorragend in ein Arbeitsumfeld integrierten, das durch ein hohes Maß kollegialer Solidarität sowie Freundschaft gekennzeichnet war. Formal wiesen mich der Kanzler und Botschafter den Bereichen Kultur sowie Presse und Öffentlichkeitsarbeit zu, so dass sich ausreichend Schnittmengen mit den Vorgaben des Islambeobachtererlasses ergaben. Auch die spezifische Situation in Tadschikistan erwies sich als eine interessante Herausforderung. Tadschikistans Entwicklung nach der unerwarteten Unabhängigkeit 1991 ist überschattet durch einen verheerenden Bürgerkrieg (1992-1997)

34 Im spezifischen Fall Tadschikistans existierte eine Agentur für religiöse Angelegenheiten (bis Januar 2007), die von staatlicher Seite islamische Belange reglementierte. Die derzeitige tadschikische Regierung verfolgt eine äußerst repressive Politik gegenüber Ausdrucksformen islamischer Religiosität mit einem ostentativen Verweis auf den säkularen Charakter der Verfassung. Während der Sowjetzeit haben sich zudem verschiedene Gruppen islamischer Würdenträger herausgebildet, die vereinfacht als ,offizieller' und ,inoffizieller' Islam bezeichnet werden können; vgl. Oliver Roy: The New Central Asia. The Creation of Nations, London: Tauris 2000 und Adeeb Khaleed: Islam after Communism. Religion and Politics in Central Asia, Berkeley: University of California Press 2007. 
und ein fragiles Friedensabkommen, das eine islamische politische Partei zeitweilig in die Regierung integrierte. ${ }^{35}$ Obwohl der ,Islam ' in Zentralasien zumeist als intellektuell wenig anspruchsvoll und institutionell schwach ausgeprägt beschrieben wird, ${ }^{36}$ entwickelte sich in Tadschikistan bereits während der sowjetischen Zeit ein komplexes Geflecht aus offiziellen und informellen islamischen Strukturen sowie Identitätsmustern - doch dies geht über die Grenzen des vorliegenden Beitrags hinaus. ${ }^{37}$

\section{Ein gut gemeinter Dialog?}

Die Konzeption des ,Islamdialogs' seitens des Auswärtigen Amtes zeigt deutlich das Bemühen, ,Islam“ weder essentialistisch noch entlang notorischer Bedrohungsszenarien $\mathrm{zu}$ thematisieren. Auch ist die heutige auswärtige Politik weit entfernt von den ambitionierten und bizarren Vorstellungen einer ,Islam- ' oder ,Orientpolitik', wie sie im Vorfeld und während des Ersten Weltkrieges von deutschen Diplomaten und Orientalisten erträumt wurde. Allerdings spiegelt die Idee des Dialogs der Kulturen bzw. des Dialogs mit der islamischen Welt eine performative Arroganz wieder, denn:

,[t]atsächlich verfestigt das Paradigma des Dialogs der Kulturen genau jene manichäische Wahrnehmung, gegen das es sich scheinbar wendet: Als ob es bei Islam und Westen um zwei Subjekte handelte, die sich nun endlich verstehen müssten. [...] Nein, der Dialog der Kulturen ist eine Karikatur, nicht weniger als der Kampf der Kulturen. Das Problem ist allerdings, dass sich diese Karikaturen in immer mehr Köpfen festsetzen und dann zu politischem oder gar militärischem Handeln führen. Am 11. September sollte offenkundig im Namen ,des' Islams, der' Westen bekämpft werden statt eines konkreten Staats oder einer Regierung, wie Terroristen es bislang vorgaben. Aber auch in Europa wird die eigene Kultur zunehmend essentialisiert, also als eine eigenständige

35 Obwohl der Bürgerkrieg gelegentlich als ein Konflikt zwischen islamischen und post-kommunistischen Fraktionen dargestellt wird, waren es vor allem regionale Identitäten, sozial-ökonomische Faktoren und der Einfluss von Strukturen organisierten Verbrechens, die die wesentlichen Ursachen des Konfliktes darstellen (vgl. O. Roy, New Central Asia, passim). Eine konzise Darstellung des tadschikischen Bürgerkrieges steht allerdings noch aus.

36 Vgl. etwa T. Jeremy Gunn: „Shaping an Islamic Identity: Religion, Islamism, and the State in Central Asia", in: Sociology of Religion 64 (2003), S. 389-410; Vitalij V. Naumkin: Radical Islam in Central Asia: Between Pen and the Rifle (Soviet Bloc and After), Lanham u.a.: Rowman \& Littlefield 2005 oder Martha Brill Olcott: Roots of Radical Islam in Central Asia. (Carnegie Papers, 77), Washington 2007.

37 Vgl. die Überlegungen in: Robert Hefner: „Public Islam and the Problem of Democratization", in: Sociology of Religion 62 (2001), S. 491-514. Für Zentralasien siehe vor allem International Crisis Group (ICG): Central Asia: Islam and the State (ICG Asia Report, 59), Osh, Brussels 2003, sowie idem: Radical Islam in Central Asia: Responding to Hizb ut-Tahrir (ICG Asia Report, 58), Osh, Brussels 2003. 
anthropologische Größe gedacht, die unabhängig vom Menschen existiert und handelt." ${ }^{\text {38 }}$

Betrachtet man die Dialogidee des Auswärtigen Amtes stellt sich letztendlich die Frage, welches die Indikatoren für einen erfolgreichen, Islamdialog ${ }^{\text {' }}$ sind.

Öffentlichkeitsarbeit, die Einladung islamischer Würdenträger zu diversen Konferenzen und Seminaren, Austausch von Jugendlichen oder die Einbeziehung islamischer Gruppierungen in politische Prozesse sind Initiativen, deren Resultate erst mittel- oder langfristig einzuschätzen sind - und dies ist vielleicht das eigentliche Problem der Konzeption des Auswärtigen Amtes. Der auswärtige Dienst untersteht dem Paradigma des Generalismus, d.h. Beamten des auswärtigen Dienstes sollen in allen Bereichen des Auswärtigen Amtes einsetzbar sein, wobei die Standzeiten von Diplomaten in bestimmten Bereichen sowie Auslandsvertretungen in der Regel 3 bis 4 Jahre betragen. Eine Spezialisierung wird im diplomatischen Dienst bis auf wenige Ausnahmen weder unterstützt noch ermutigt, auch der Bildungsweg spielt nur eine untergeordnete Rolle - ein Erbe aus der Frühzeit des Auswärtigen Amtes, in der Loyalitätskonflikte im Falle von langen Standzeiten oder Spezialisierungen antizipiert wurden. Die Herausforderungen an die Außenpolitik nach dem 11. September 2001 und vielleicht auch die Personalpolitik im Rahmen der ATP führte zu einer internen Diskussion im Auswärtigen Amt, inwieweit Spezialisierungen (und damit längere Standzeiten bzw. Konzentration auf bestimmte Aufgabengebiete) im auswärtigen Dienst eine sinnvolle Ergänzung zu der bestehenden Personalpolitik darstellen könnten. Die Reforminitiative wurde im Frühjahr 2004 zurückgewiesen. Letztendlich hatte dies zur Folge, dass die ,Islambeobachtung 'vor allem in den Auslandsmissionen nach zwei oder drei Jahren aufgrund von Versetzung bzw. Vertragsende entweder endete oder zumindest unterbrochen wurde. Angesichts der mangelnden Kontinuität ist es zweifelhaft, ob der ,Islamdialog ' mittel- oder langfristige Auswirkungen zeitigen wird. Die Vermutung liegt nahe, dass das Auswärtige Amt den ,Islamdialog ' vor allem als eine Chance in der Personalpolitik sah, Planstellen zu schaffen, die in den Jahren zuvor finanziellen Zwängen zum Opfer gefallen waren.

\section{Das Für und Wider einer angewandten Orientalistik}

Die Frage nach der gesellschaftlichen Verantwortung von Wissenschaft ist von ungebrochener Aktualität, dies gilt nach dem 11. September insbesondere für die Orientalistik, wobei diese Verantwortung zwei Ebenen hat: Zum einen betrifft sie das Verhältnis zum politischen Feld und zum anderen die Ebene des spezifischen Diskurses (d.h. der ,Repräsentation“ im Sinne Saids) über den islamischen Kul-

38 N. Kermani: Zur Zukunft der Islamwissenschaft, S. 8. 
turraum. Die öffentliche Debatte über ,Islam" wird auf absehbare Zeit ihre hohe Relevanz bewahren und die Orientalistik herausfordern - dies betrifft vor allem die vermeintlich ,angewandte Orientalistik' mit unmittelbaren politischen Bezügen. Der so genannte ,Krieg gegen den Terror" mit all seinen diffusen Gefahrendiskursen, Doppelstandards und rechtlichen Grauzonen (bis hin zu gravierenden Verletzungen essentieller Menschenrechte) ist in diesem Zusammenhang vielleicht die größte Herausforderung, da zunehmend auch Orientalisten auf verschiedenen Ebenen involviert sind. Dies schließt nicht nur die Wortführer eines Zivilisationskonfliktes ein, die aus der sicheren Distanz ihrer Washingtoner Büros eine vermeintlich ,sachkundige Islampolitik' propagieren, sondern zunehmend auch Mitarbeiter staatlicher wie nicht-staatlicher Institutionen, die with boots on the ground politisch motivierte ,Islamprojekte 'implementieren. ${ }^{39}$

Die gegenwartsbezogene Beschäftigung mit der islamischen Welt bedeutet vor allem auch die Auseinandersetzung mit überwiegend säkularen autoritären Staaten, die die Menschenrechte ihrer Bürger massiv verletzen, aber aufgrund politisch-militärischer Erwägungen im ,Krieg gegen den Terror ' von den USA oder den EU-Staaten hofiert werden. Die Verantwortungsethik der Wissenschaft wird hier gleich in zweierlei Hinsicht strapaziert: Zum einen in Bezug auf das ,Forschungsobjekt', d.h. wie führt man in einem autoritären politischen System Forschungsprojekte durch, ohne dass man entweder Kollegen oder Kontaktper-

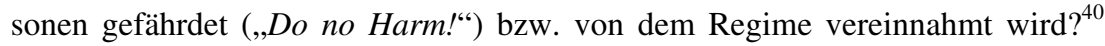

39 Neben Deutschland verfolgen auch die USA und Großbritannien so genannte „Muslim Outreach" Projekte, die im Bereich der Öffentlichkeitsarbeit angesiedelt sind. Einige dieser Projekte zielen jedoch auf eine direkte Einflussnahme auf Gesellschaften in islamischen Ländern ab bzw. haben eine militärische, sicherheitsorientierte Komponente. Das US State Department stellt in diesem Zusammenhang umfangreiche Finanzmittel für eine ,Demokratisierung ' bzw. einen Regimewechsel in Iran zur Verfügung. Die New York Times berichtete am 5.10.2007 (David Rohde: „Army Enlists Anthropolgy in War Zones"), dass US-Truppen in Afghanistan von Zivilisten mit einschlägiger Ausbildung in Anthropologie und Landeskunde begleitet werden.

40 Usbekistan ist in diesem Zusammenhang ein trauriges Beispiel, insbesondere hinsichtlich der Niederschlagung des Aufstandes in Andijan im Mai 2005 mit vermutlich Hunderten von getöteten Zivilisten. Eine Reihe von gut dokumentierten Berichten bemüht sich um eine Rekonstruktion der Ereignisse in Andijan vgl. etwa Human Rights Watch: Bullets Were Falling Like Rain. The Andijan Massacre, May 13, 2005; New York, Juni 2005; ICG: Uzbekistan: The Andijon Uprising (Asia Briefing No. 38); Bischkek, Brüssel 2005; Organisation for Security and Co-operation in Europe (OSCE)/Office of Democratic Institutions and Human Rights (ODIHR): Preliminary Findings on the Events in Andijan, Uzbekistan, 13 May 2005; Warschau 2005. Während usbekische und russische Medien die Niederschlagung des Aufstandes als einen Sieg im Krieg gegen den Terror feierten, war die westliche Berichterstattung gekennzeichnet von Schock und Abscheu. Das usbekische Regime fand nur wenige Fürsprecher, unter denen allerdings Shirin Akiner vom SOAS die vielleicht lautstärkste ist. Akiner besuchte Andijan 12 Tage nach dem gescheiterten Aufstand in Begleitung hochrangiger Vertreter des usbekischen Regimes und ver- 
Zum anderen in Bezug auf die politische Agenda, d.h. inwieweit steht insbesondere die orientalistische Forschung im Dienst des ,Kriegs gegen den Terror“. Diesen Herausforderungen muss sie sich stellen.

fasste einen Bericht, der sich kritisch mit der westlichen Berichterstattung auseinandersetzt und die Sichtweise des usbekischen Regimes unterstützt (vgl. Shirin Akiner: Violence in Andijan, 13 May 2005: An Independent Assessment; Silk Road Paper, 2005 auf http://www.silkroadstudies.org/new/inside/publications/0507Akiner.pdf). Grundsätzlich ist an einer kritischen Auseinandersetzung mit den Medien nichts auszusetzen, allerdings ist die apologetische Darstellung von Akiner extrem einseitig und verzichtet gänzlich auf eine Einordnung der Ereignisse in einen breiteren Kontext. Insbesondere ihre enge Kollaboration mit Vertretern des usbekischen Regimes (inklusive eines Interviews im usbekischen Staatsfernsehen) ist im Sinne einer Wissenschaftsethik höchst problematisch. 